\title{
Cortical Control of Hering-Breuer Reflexes in ANESTHETIZED RATS
}

\author{
V. G. Aleksandrov¹, V. A. Mercuriev¹, T. G. Ivanova ${ }^{1}$, A. A. Tarasievich ${ }^{1}$, N. P. Aleksandrova²
}

\author{
${ }^{1}$ Department of Human and Animal Anatomy and Physiology, Herzen State Pedagogical University of Russia, St. Petersburg, Russia; \\ ${ }^{2}$ Laboratory of Respiration Physiology, Pavlov Institute of Physiology RAS, St. Petersburg, Russia
}

\begin{abstract}
It had been hypothesized that the regions of prefrontal cortex which are involved in respiratory control can modulate Hering-Breuer reflexes evoked by vagal input from pulmonary stretch receptors. In the present study, experiments were performed on urethane anesthetized spontaneously breathing Wistar rats. The expiratory-promoting reflex was evaluated from changes in expiratory time immediately after airway occlusion at the end of inspiration. The inspiratory-inhibitory reflex was estimated from changes in inspiratory time provoked by airway occlusion at the end of expiration. The results indicate that electrical microstimulation of the responsive sites within the insular cortex significantly weakened both expiratory-promoting and inspiratory-inhibitory reflex. Activation of the infralimbic cortex depressed expiratory-promoting reflex, but inspiratory-inhibitory reflex was enhanced. These results suggest that stimulation of the prefrontal cortex influences vagally mediated control of the respiratory phases timing and several regions of the prefrontal cortex modulate distinct sets of neurons in the network controlling inspiratory and expiratory phases of a breath cycle.
\end{abstract}

Key words: prefrontal cortex, microstimulation, Hering-Breuer reflex

\section{INTRODUCTION}

Prefrontal cortex (PFC) has long been implicated in autonomic control playing a key role in the coordination of affective and autonomic responses $[1,2]$. Dysfunction of PFC can lead to disturbances in autonomic regulation and neuroendocrine responses that are associated with mood disorders [3]. An increasing knowledge of associations between PFC lesions and stroke-induced executive deficits, such as cardiac complications and respiratory dysfunction [4-8], stimulates interest concerning the role of the central autonomic nervous system in cardiovascular and respiratory control. It has been found that there is a distinct interaction of the insular cortex (IC, lateral PFC) with heart rate and blood pressure control [9-11]. Our previous studies have confirmed that IC and infralimbic cortex (IL, ventromedial PFC) are involved in the respiratory control, because electric stimulation of these areas produced distinct patterns of respiratory responses $[12,13]$. However, it remains an unresolved issue what mechanisms mediate PFC influences on the autonomic control. It is suggested that PFC influence on the cardiovascular control is mediated by the parasympathetic activity, because inactivation of the ventromedial PFC withdraws parasympathetic input to the baroreflex [14]. It is also established that stimulation of the IL cortex inhibits vagally mediated relaxation of the stomach [15]. Therefore, it seems that PFC exerts autonomic function by modulating the reflexes mediated by vagal afferents. A substantial part of vagal afferents originates from slow adapting pulmonary stretch receptors (SARs) which induce Hering-Breuer reflexes. Therefore, in the present study we investigated the hypothesis that stimulation of both IC and IL cortices will influence the Hering-Breuer reflexes.

\section{Material AND Methods}

All animal procedures were conducted in accordance with the ethical guidelines of the European Community Council Directives 86/609/EEC and the study was approved by a local Ethics Committee. Experiments were done in 23 spontaneously breathing male Wistar rats weighing 250-300 $\mathrm{g}$ which were anaesthetized with urethane $\left(1.25 \mathrm{~g} \cdot \mathrm{kg}^{-1}\right.$, i.p.). Anesthesia level was sufficient to eliminate pain reflexes and animal did not response to noxious stimuli (tactile pinches). Rectal temperature was monitored and maintained at $37 \pm 0.5^{\circ} \mathrm{C}$ with a thermal controller (ML295/R; ADInstruments Pty, Bella Vista NSW, Australia). The animals were tracheostomized and canulated with a respiratory flow head (MLT1L; ADInstruments Pty) which was connected to a pneumotachometer (ML141 Spirometer; ADInstruments Pty). Esophageal pressure $\left(\mathrm{P}_{\mathrm{es}}\right)$ was measured in the lower third of esophagus with an airfilled latex balloon connected to a custom-made low pressure transducer. A cannula filled with heparinized saline was inserted into the femoral artery for recording of arterial blood pressure with a blood pressure transducer (MLT0670; ADInstruments Pty). Pressure signals were fed into the bridge amplifier (ML224; ADInstruments Pty). Pneumotachometer and bridge amplifier were coupled with a data acquisition system 
(PowerLab 8/30; ADInstruments Pty) supplied with a conventional software. The rats were placed in a stereotaxic frame in the prone position, with the head fixed in the 'flat scull' position. Electrostimulation of the cortex was made using a lacquer insulated tungsten electrode with a tip diameter of $50 \mu \mathrm{m}$. Electrode was immersed into the hemisphere with steps of $250 \mu \mathrm{m}$ by means of a stereotactic head. A trial stimulus (5-10 $\mathrm{s}$ train of negative $0.5 \mathrm{~ms}$ pulses at $60 \mathrm{~Hz}$ and $100 \mu \mathrm{A}$ ) was applied after each step to detect respiratory responses. Then, the electrode was left set in the most sensitive site and the experimental current strength was set at $50 \%$ above the threshold level.

After surgery, animals were allowed $30 \mathrm{~min}$ for stabilization. The pneumotachogram, $\mathrm{P}_{\mathrm{es}}$ and arterial blood pressure were continuously recorded. The experiment started with a recording of the respiratory response to cortical stimulation and the effects of expiratory and inspiratory occlusions were tested afterwards. Then, the stimulation current was switched on again and, after 2-3 breaths, airway occlusion was superimposed on cortical stimulation. The above protocol was repeated several times before the electrode was moved to another cortical site. At the end of the experiment, an electrolytic lesion was made at the bottom of the last electrode track. The animal was killed with an overdose of urethane and perfused with 100 $\mathrm{ml}$ of $0.9 \%$ saline followed by $250 \mathrm{ml}$ of $10 \%$ formalin solution. Cortical tissue specimens were prepared and histological verification of the stimulated sites was performed as previously described [13].

Pneumotachography was used for measuring maximal inspiratory flow $\left(\mathrm{V}_{\text {Imax }}\right)$, tidal volume $(\mathrm{VT})$, inspiratory time $\left(\mathrm{T}_{\mathrm{I}}\right)$, and expiratory time $\left(\mathrm{T}_{\mathrm{E}}\right)$. Inspiratory swings of esophageal pressure were recorded to estimate total inspiratory effort $\left(\mathrm{P}_{\mathrm{es}}\right)$ and to approximate Ti and Te during airway occlusion. Airway occlusions were performed either at end-inspiration or at end-expiration. Expiratory occlusions were used for the purpose of estimating the strength of the Hering-Breuer inspiratory-inhibitory (TI-inhibitory) reflex, whereas inspiratory occlusions enabled to assess the HeringBreuer expiratory-promoting (TE-promoting) reflex. In order to obtain quantitative characteristics of these reflexes, the absolute values of $\mathrm{T}_{\mathrm{I}}$ or $\mathrm{Te}$ were estimated in the first post-occlusive breath and expressed as a percent of the corresponding last pre-occlusive breath. The magnitude of these indexes was taken as reflecting the strength of the reflexes. Statistical analyses were performed using the non-parametric Wilcoxon signed-rank test. $\mathrm{P}<0.05$ were considered significant.

\section{RESULTS}

The results demonstrate that the same stimulus train applied to the infralimbic or insular cortex altered the pattern of breathing in a different way. The responsive sites in the IL cortex were located between 1.5-2.5 mm rostral to bregma at a depth of $4.5-5.0 \mathrm{~mm}$ from the brain surface. The IL responses consisted of a progressive decrease of $\mathrm{T}_{\mathrm{T}}$ and $\mathrm{V}_{\mathrm{T}}$ (Fig. 1A). In the first breath after the start of stimulation, $\mathrm{T}_{\mathrm{T}}$ and $\mathrm{V}_{\mathrm{T}}$ fell to $58.0 \pm 4.7 \%$, and $70.0 \pm 7.7 \%$ of the control level, re- spectively. The changes stabilized during the successive 3-4 breath and remained so until the end of the stimulus period $(\mathrm{P}<0.05)$. Stimulation of the responsive sites in the IC cortex $0.5-1.5 \mathrm{~mm}$ rostral to bregma produced inhibitory responses manifested in a decrease of $\mathrm{V}_{\text {Imax }}, \mathrm{V}_{\mathrm{T}}$, and $\mathrm{P}_{\mathrm{es}}$ (Fig. 1B). In the first breath, $\mathrm{V}_{\mathrm{Imax}}, \mathrm{V}_{\mathrm{T}}$, and $\mathrm{P}_{\mathrm{es}}$ decreased to $53.0 \pm 5.6 \%$, $43.0 \pm 3.6 \%$, and $57.0 \pm 5.4 \%$ of the control level, respectively $(\mathrm{P}<0.05)$. The magnitude of the decreases tapered off in the successive respiratory cycles during the stimulation period, until the variables returned to the control levels. Therefore the IC responses were transient character.

During control conditions, airway occlusion at endinspiration prolonged $\mathrm{T}_{\mathrm{E}}$ and increased $\mathrm{P}_{\mathrm{es}}$ in the first and following breaths, but the $\mathrm{T}_{\mathrm{E}}$ of the first expiration was prolonged to a greatest extent (Fig. 2A-a). The $\mathrm{Te}_{\mathrm{E}}$ increased by $500-700 \%$ of the control value. Airway occlusion at end-expiration evoked a prolongation of $\mathrm{T}$ i and an increase of $\mathrm{P}_{\mathrm{es}}$ in the first and following occluded breaths (Fig. 2B-a). Within the first occluded inspiration, the increment of the inspiratory time was $170-250 \%$ of the control level. Stimulation in the prefrontal cortex modified the effects of occlusions. In particular, IL and IC stimulations shortened Te which was already prolonged by inspiratory occlusion (Fig. 2A-b, c). On the other side, stimulation of the IL enhanced the effect of expiratory occlusion which manifested itself in the increase in Ti (Fig. 2Bb). Stimulation of the IC had a similar effect on $\mathrm{TI}_{\mathrm{I}}$ (Fig. 2B-c). Further analysis permitted to make more definite conclusions about the effects of the stimulation of either prefrontal cortex exerted on the HeringBreuer reflexes. It was confirmed that stimulation of IL, just as that of IC, significantly weakened the TEpromoting reflex (Fig. 3A). As to the TI-inhibitory reflex, IL promoted and IC depressed it, but the latter effect turned out to be insignificant (Fig. 3B).

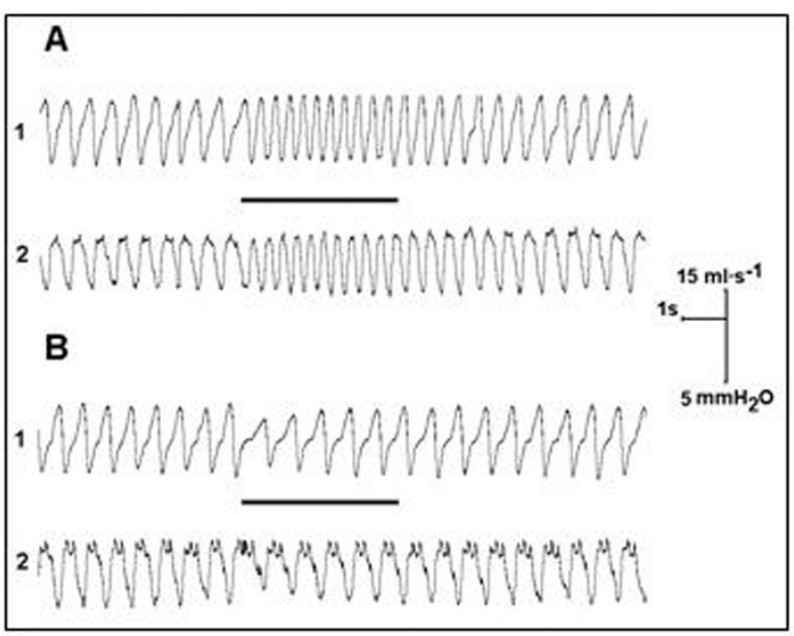

Fig. 1. Changes in breathing pattern during electrical stimulation of different prefrontal areas.

A - infralimbic cortex (IL); B - anterior part of insular cortex (IC); 1 - respiratory flow and 2- inspiratory swings of esophageal pressure. Horizontal lines indicate duration of stimulation consisting of $5 \mathrm{~s}$ train of $0.5 \mathrm{~ms}$ pulses at $60 \mathrm{~Hz}$ and $150 \mathrm{~mA}$. 

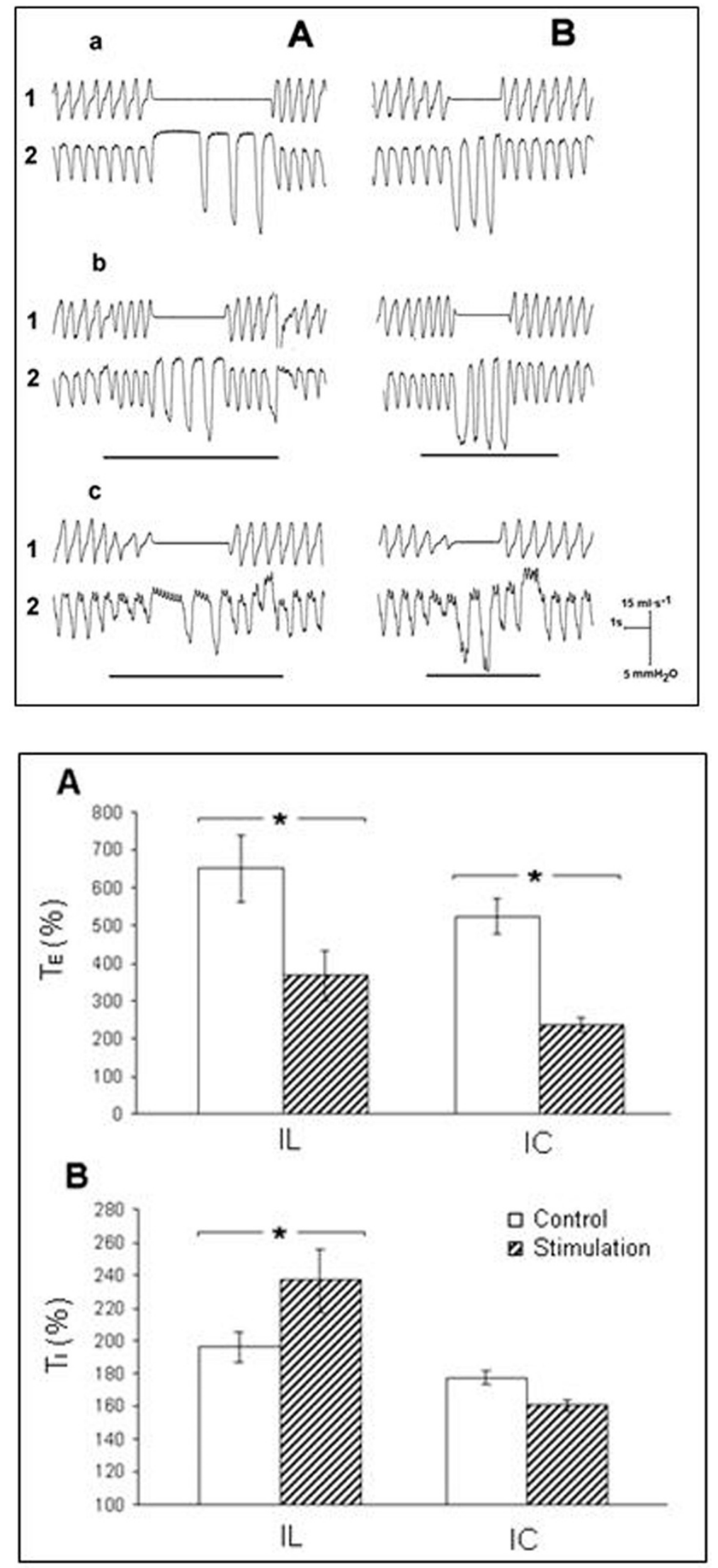

Fig. 2. Influence of cortical stimulation on the effects of airway occlusions. A - occlusions at the end-inspiration and B - occlusions at end-expiration; $\mathrm{a}$ - control, $\mathrm{b}$ - stimulation of infralimbic cortex (IL), and c - stimulation of insular cortex (IC); 1 - respiratory flow and 2 - inspiratory swings of esophageal pressure. Stimulus characteristics are the same as in Fig.1. Horizontal lines indicate duration of stimulation.

Fig. 3. Alterations in Hering-Breuer reflexes in response to cortical stimulation. A - expiratorypromoting reflex; B - inspiratory-inhibitory reflex. Te $(\%)$ - duration of the first expiration after inspiratory occlusion expressed as a percent of the TE of the last pre-occluded breath; Ti (\%) - duration of the first inspiration after expiratory occlusion expressed as a percent of the Ti of the last pre-occluded breath. $* \mathrm{P}<0.05$.

\section{Discussion}

In agreement with our previous studies [12, 13], the present study demonstrate that distinct patterns of respiratory responses localize to different parts of the prefrontal cortex in the rat. The present findings show that stimulation of different PFC fields produced specific alterations in the respiratory pattern. 
The SARs are activated by lung inflation and afferent input descending from SARs is important for the timing of respiratory phases. Afferent traffic from SARs delivered during inspiration terminates inspiratory phase [16], and that during expiration prolongs the expiratory period [17]. These reactions are referred to as Hering-Breuer inspiratory-inhibitory (TI-inhibitory) and Hering-Breuer expiratory-promoting (TE-promoting) reflex, respectively. If the airway occlusion is performed at end-expiration, lung volume remains at the level of functional residual capacity. Afferent traffic from SARs is minimal under such conditions, therefore, the TI-inhibitory reflex weakens and TI of the immediately following inspiration increases. On the other hand, airway occlusion at end-inspiration keeps afferent traffic high and increases the strength of the Te-promoting reflex. Therefore, it is obvious that both TI-inhibitory and TE-promoting reflex were evoked in the present study.

This study describes a novel phenomenon which consists of alterations in the Hering-Breuer reflexes in response to electrical microstimulation applied to sites in the prefrontal cortex. This fact suggests that the prefrontal cortex is able to modulate respiratory reflexes evoked by SARs. The present study indicates that stimulation of the IC weakens both TE-promoting and $\mathrm{T}_{\mathrm{I}}$ - inhibitory reflexes, and activation of the IL depresses the TE-promoting reflex. Thus, prefrontal cortex effects on the Hering-Breuer reflexes are inhibitory for the most part. This fact is in accordance with the results of the previous studies which have demonstrated an inhibitory action of the prefrontal cortex on autonomic reflexes $[14,15]$. In the present study, however, the IL increased the strength of the TI-inhibitory reflex, which suggests that PFC effects on the autonomic reflexes may be excitatory.

It seems clear that the anatomic substrate for this cortical action on respiratory reflexes is formed by extensive direct projections from the PFC to the nucleus tractus solitarius (NTS) [18-22]. The NTS is the main brainstem recipient of vagal afferents which conduct viscerosensory information from various viscera, including lungs, and a number of autonomic reflexes, including Hering-Breuer reflexes, integrate within the NTS [23-25]. Therefore, PFC may influence transmission through the various reflex pathways which starts with the vagal afferents and switches in the NTS onto the neurons of the dorsal respiratory group. Besides, respiratory neurons mediating the Hering-Breuer TEpromoting reflex have been identified in the ventral respiratory group (VRG) situated in the ventrolateral medulla [26]. The ventrolateral medulla, in turn, receives afferents from the PFC and other autonomic structures related with it $[22,27]$. Therefore, PFC effects on the Hering-Breuer reflexes may be mediated by neurons of the DRG and VRG.

In summary, the present study demonstrates that impulses originating from the prefrontal cortex can influence the vagally-mediated control of the respiratory phase timing mechanism. It is suggested that several regions in the prefrontal cortex modulate distinct sets of neurons in the network controlling inspiratory and expiratory phases. This mechanism could play an important role in the cortical control of breathing pat- terns associated with emotional and stressful behavioral disturbances, such as dyspnea or breathlessness.

Conflicts of interest: No conflicts of interest were declared by the authors in relation to this article.

\section{REFERENCES}

[1] Hänsel A, von Känel R. The ventro-medial prefrontal cortex: a major link between the autonomic nervous system, regulation of emotion, and stress reactivity? Biopsychosoc Med 2008; 2: 21.

[2] Barbas H, Saha S, Rempel-Clower N, Ghashghaei T. Serial pathways from primate prefrontal cortex to autonomic areas may influence emotional expression. BMC Neurosci 2003; 4: 25

[3] Drevets WC, Price JL, Furey ML. Brain structural and functional abnormalities in mood disorders: implications for neurocircuitry models of depression. Brain Struct Funct 2008; 213(1-2): 93-118.

[4] Davenport RJ, Dennis MS, Wellwood I, Warlow CP. Complications after acute stroke. Stroke 1996; 27: 415- 20.

[5] Johnston KC, Li JY, Lyden PD, Hanson SK, Feasby TE, Adams RJ, Faught RE Jr, Haley EC Jr. Ranttas investigators. Medical and neurological complications of ischemic stroke: experience from the Ranttas trial. Stroke 1998; 29: 447- 53.

[6] Kocan MJ. The brain-heart connection: cardiac effects of acute ischemic stroke. J Cardiovasc Nurs 1998; 13: 57 68.

[7] Rochester CL, Mohsenin V. Respiratory complications of stroke. Semin Respir Crit Care Med 2002; 23: 248 -60.

[8] Dutsch M, Burger M, Dorfler C, Schwab S, Hilz MJ. Cardiovascular autonomic function in post stroke patients. Neurology 2007; 69: 2249-55.

[9] Oppenheimer SM, Cechetto DF, Hachinski VC. Cerebrogenic cardiac arrhythmias: cerebral electrocardiographic influences and their role in sudden death. Arch Neurol 1990; 47: 513-19.

[10] Oppenheimer SM, Wilson JX, Guiraudon C, Cechetto DF. Insular cortex stimulation produces lethal cardiac arrhythmias: a mechanism of sudden death? Brain Res 1991; 550: 115-21.

[11] Oppenheimer S. Cerebrogenic cardiac arrhythmias: cortical lateralization and clinical significance. Clin Auton Res 2006; 16: 6-11.

[12] Aleksandrov VG, Aleksandrova NP, Bagaev VA. Identification of a respiratory related area in the rat insular cortex. Can J Physiol Pharmacol 2000; 78(7): 582-86.

[13] Alexandrov VG, Ivanova TG, Alexandrova NP. Prefrontal control of respiration. J Physiol Pharmacol 2007; 58 Suppl 5: 17-23.

[14] .Resstel LB, Fernandes KB, Correa FM. Medial prefrontal cortex modulation of the baroreflex parasympathetic component in the rat. Brain Res 2004; 1015(1-2): 136-44.

[15] Panteleev S, Grundy D. Descending influences from the infralimbic cortex on vago-vagal reflex control of gastric motor activity in the rat Auton Neurosci 2000; 86(1-2): $78-83$.

[16] Cohen MI, Feldman JL. Discharge properties of dorsal medullary inspiratory neurons: relation to pulmonary afferent and phrenic efferent discharge. J Neurophysiol 1984; 51(4): 753-76.

[17] Grippi MA, Pack AI, Davies RO, Fishman AP. Adaptation to reflex effects of prolonged lung inflation. J Appl Physiol 1985; 58(4): 1360-71.

[18] Van der Kooy D, Koda LY, McGinty JF, Gerfen CR, Bloom FE. The organization of projections from the cor- 
tex, amygdala, and hypothalamus to the nucleus of the solitary tract in rat. J Comp Neurol 1984; 224(1): 1-24

[19] Terreberry RR, Neafsey EJ. The rat medial frontal cortex projects directly to autonomic regions of the brainstem. Brain Res Bull 1987; 19(6): 639-49.

[20] Cechetto DF, Saper CB. Role of cerebral cortex in autonomic functions. In The autonomic nervous system: Central regulation of autonomic functions. Oxford University Press, 1990; pp. 208-33.

[21] Hurley KM, Herbert H, Moga MM, Saper CB. Efferent projections of the infralimbic cortex of the rat. J Comp Neurol 1991; 308(2): 249-76.

[22] Yasui Y, Breder CD, Saper CB, Cechetto DF. Autonomic responses and efferent pathways from the insular cortex in the rat. J Comp Neurol 1991; 303(3): 355-74.

[23] Kalia M, Mesulam MM. Brain stem projections of sensory and motor components of the vagus complex in the cat: II. Laryngeal, tracheobronchial, pulmonary, cardiac, and gastrointestinal branches. J Comp Neurol 1980; 193(2): 467-508.

[24] Bonham AC, McCrimmon DR. Neurones in a discrete region of the nucleus tractus solitarius are required for the Breuer-Hering reflex in rat. J Physiol 1990; 427: 261 80
[25] Lipski J, Ezure K, Wong She RB. Identification of neurons receiving input from pulmonary rapidly adapting receptors in the cat. J Physiol 1991; 443: 55-77.

[26] Hayashi F, Coles SK, McCrimmon DR. Respiratory neurons mediating the Breuer-Hering reflex prolongation of expiration in rat. J Neurosci 1996; 16(20): 6526-36

[27] Gabbott PL, Warner TA, Jays PR, Salway P, Busby SJ Prefrontal cortex in the rat: projections to subcortical autonomic, motor, and limbic centers. J Comp Neurol 2005; 492(2): 145-77.

Corresponding author:

Vjacheslav G. Aleksandrov

Department of Human and Animal Anatomy and Physiology Herzen State Pedagogical University of Russia

St. Petersburg, Russia

E-mail:vvg_aleks@yahoo.com 